\title{
Correction to: Human Development Index and its association with staff spiritual care provision: a Middle Eastern oncology study
}

Gil Bar-Sela ${ }^{1}$ (D) Michael J. Schultz ${ }^{2} \cdot$ Karima Elshamy $^{3} \cdot$ Maryam Rassouli $^{4} \cdot$ Eran Ben-Arye $^{5} \cdot$ Myrna Doumit $^{6}$. Nahla Gafer ${ }^{7}$. Alaa Albashayreh ${ }^{8}$. Ibtisam Ghrayeb ${ }^{9}$. Ibrahim Turker ${ }^{10}$. Gulcin Ozalp ${ }^{10}$. Sultan Kav ${ }^{11}$. Rasha Fahmi ${ }^{12}$. Sophia Nestoros ${ }^{13}$. Hasanein Ghali ${ }^{14}$. Layth Mula-Hussain ${ }^{15}$. Ilana Shazar ${ }^{16}$. Rana Obeidat ${ }^{17}$.

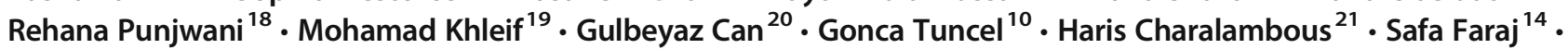
Neophyta Keoppi ${ }^{22} \cdot$ Mazin Al-Jadiry ${ }^{14} \cdot$ Sergey Postovsky ${ }^{23} \cdot$ Ma'an Al-Omari $^{24} \cdot$ Samaher Razzaq $^{14} \cdot$ Hani Ayyash $^{25}$. Khaled Khader ${ }^{26} \cdot$ Rejin Kebudi $^{27}$. Suha Omran ${ }^{28}$. Osaid Rasheed ${ }^{29} \cdot$ Mohammed Qadire $^{30} \cdot$ Ahmet Ozet $^{31}$. Michael Silbermann ${ }^{32}$

Published online: 24 April 2019

(C) Springer-Verlag GmbH Germany, part of Springer Nature 2019

Correction to: Supportive Care in Cancer https://doi.org/10.1007/s00520-019-04733-0

The name of Layth Mula-Hussain was incorrectly captured in the original manuscript.

The original article has been corrected.

Publisher's note Springer Nature remains neutral with regard to jurisdictional claims in published maps and institutional affiliations.

The online version of the original article can be found at https://doi.org/ 10.1007/s00520-019-04733-0

Gil Bar-Sela

gil_ba@clalit.org.il

Extended author information available on the last page of the article 


\section{Affiliations}

\section{Gil Bar-Sela ${ }^{1}$ (D) Michael J. Schultz ${ }^{2}$ - Karima Elshamy ${ }^{3} \cdot$ Maryam Rassouli $^{4} \cdot$ Eran Ben-Arye $^{5} \cdot$ Myrna Doumit $^{6}$. Nahla Gafer ${ }^{7}$. Alaa Albashayreh ${ }^{8} \cdot$ Ibtisam Ghrayeb $^{9} \cdot$ Ibrahim Turker $^{10} \cdot$ Gulcin Ozalp $^{10} \cdot$ Sultan Kav $^{11}$. Rasha Fahmi ${ }^{12}$. Sophia Nestoros ${ }^{13}$. Hasanein Ghali ${ }^{14}$ - Layth Mula-Hussain ${ }^{15}$ • Ilana Shazar ${ }^{16}$. Rana Obeidat $^{17}$. Rehana Punjwani $^{18}$. Mohamad Khleif ${ }^{19} \cdot$ Gulbeyaz Can $^{20} \cdot$ Gonca Tuncel $^{10} \cdot$ Haris Charalambous $^{21} \cdot$ Safa Faraj $^{14}$. Neophyta Keoppi ${ }^{22}$ - Mazin Al-Jadiry ${ }^{14}$. Sergey Postovsky ${ }^{23} \cdot$ Ma'an Al-Omari $^{24} \cdot$ Samaher Razzaq $^{14}$. Hani Ayyash $^{25}$. Khaled Khader $^{26}$. Rejin Kebudi ${ }^{27}$. Suha Omran ${ }^{28}$. Osaid Rasheed ${ }^{29}$ - Mohammed Qadire ${ }^{30}$. Ahmet Ozet $^{31}$. Michael Silbermann ${ }^{32}$}

Cancer Center, Emek Medical Center, Afula, Israel

2 Division of Oncology, Rambam Health Care Campus, Haifa, Israel

3 Faculty of Nursing, Mansoura University, Mansoura, Egypt

4 Cancer Research Center, Shahid Beheshti University of Medical Sciences, Tehran, Islamic Republic of Iran

5 The Oncology Service, Lin Medical Center, Haifa, Israel

6 Alice Ramez Chagoury School of Nursing, Lebanese American University, Beirut, Lebanon

7 Radiation and Isotope Center, Khartoum, Sudan

8 College of Nursing, Sultan Qaboos University, Muscat, Sultanate of Oman

9 Makassed Charitable Hospital, Bethlehem, West Bank, Palestine

10 Abdurrahman Yurtaslan Ankara Oncology Training and Research Hospital, Ankara, Turkey

11 Faculty of Health Sciences, Department of Nursing, Baskent University, Ankara, Turkey

12 El-Salam Oncology Center, Cairo, Egypt

13 Cyprus Anti-Cancer Society, Avodaphnousa Hospice, Nicosia, Cyprus

14 Children's Welfare Teaching Hospital, Baghdad College of Medicine, Baghdad, Iraq

15 Cross Cancer Institute, University of Alberta, Edmonton, Canada

16 Department of Hematology, Rambam Health Care Campus, Haifa, Israel
17 Faculty of Nursing, Zarqa University, Zarqa, Jordan

18 Children Cancer Hospital, Karachi, Pakistan

19 Al-Sadeel Society for Palliative Care, Bethlehem, West Bank, Palestine

20 Florence Nightingale Faculty of Nursing, Istanbul University, Istanbul, Turkey

21 Bank of Cyprus Oncology Center, Nicosia, Cyprus

22 Cyprus Anti-Cancer Society, Nicosia, Cyprus

23 Ruth Rappaport Children's Hospital, Rambam Health Care Campus, Haifa, Israel

24 King Abdullah University Hospital, Irbid, Jordan

25 European Khan Yunis Hospital, Khan Yunis, Gaza Strip, Palestine

26 Taif University, Taif, Saudi Arabia

27 Cerrahpasa Medical Faculty \& Oncology Institute, Istanbul University, Istanbul, Turkey

28 Faculty of Nursing, Jordan University for Science and Technology, Irbid, Jordan

29 Abu Dis and Al-Ahli Hospital, Al Quds University, Hebron, West Bank, Palestine

30 Faculty of Nursing, Al-Bayt University, Mafraq, Jordan

31 Tibbi Onkoloji Bilim Dali, Gazi Universitesi Tip Fakultesi, Ankara, Turkey

32 Middle East Cancer Consortium and Technion-Israel Institute of Technology, Haifa, Israel 